\title{
The benefits of real time data for financial market: a proposal model for banking in Albania
}

\author{
Msc.Erarda Vuka ${ }^{1,}$ Dr Julian Fejzaj ${ }^{2}$ \\ ${ }^{1,2}$ University of Tirana, Faculty of Natural Sciences, Albania
}

\begin{abstract}
.
Technology is evolving very fast and the amount of data is becoming increasingly difficult to manage, companies need to make more and more accurate decisions at the right time, hence real time data processing is taking place in any environment. Using a real-time processing model that completes transactions without delay can be the kernel of a success. Real-time data has the potential to improve the customer experience, increase office efficiency, and enhance analytics capabilities at a lower cost. Although real-time banking is not a new concept, it is one of the most difficult processes to implement in financial market, in order to get the benefits of real-time transaction processing, such as improved portfolio management, fast credit card fraud and acceptance checks, and others, banks and other financial services. Many banks have already adopted a variety of technologies and mechanisms to provide a real-time customer experience, but the implementation of these technological improvements has additional costs and difficulties that banks sometimes prefer to bypass. In this paper in the first part are presented the basic concepts of real time data, then we will look at the growing importance of real-time data flows and their impact on business and on people's lives, especially in financial services. In the final part will be presented a simple implementation of real time for banking in Albania.
\end{abstract}

Keywords: Real-time data, banking, financial market, data warehouse

\section{Introduction}

With the expansion of information technology, massive data have been generated, enterprise need to analysis data efficiently and accurately. Data warehouse, online analysis, business intelligence and data mining are developing, these technologies help enterprise to analyze data and make businesses decision. Traditional data warehouse system use historical data aggregation and analysis to provide strategic decision making, long-term planning.Many companies want to have the best decisions in time and strategy but with traditional DW this is not achieved, so today the business is moving towards RTDW.Real-time means detect and capture the changed data from business systems in time, and load data into the data warehouse. Users can access and query real-time data warehouse to make tactical decision analysis. Although over the years the benefits of real time data have been shown more and more, the 


\section{$5^{\text {th }}$ International Conference On Applied Research In SCIENCE, TECHNOLOGY \& KNOWLEDGE}

26-28 February, 2021

Amsterdam, Netherlands

solid implementation especially in finance-banking remains a challenge. Generally in banking, the dominant method of replenishing data warehouses is extract, transform and load (ETL) tools that "pull" data from source systems periodically - at the end of a day, week, or month and provide a "snapshot" of data at a given moment in time. That batch data is then loaded into a data warehouse table. During each cycle, the warehouse table is completely refreshed and the process is repeated no matter whether the data has changed or not. However if you ask customers whether today's banks already provide real-time capabilities and many will likely say "yes." After all, when you make an ATM deposit the receipt often reflects your new balance. But try confirming that new balance online! Behind ATMs, websites, and tellers, each transaction is queued up with many others and processed at a later time, usually overnight. Meanwhile, customers are seeing a temporary credit or debit (called a memo post) made to the account until the batch process is completed. Several technology advancements, increased competition, and rapidly developing customer expectations are causing many bank leaders to rethink real-time banking, and considering habits it may contribute mostly to the items on their strategic agenda, including: allow to run free product innovation; integrating customer channels (including social and mobile); improving fraud and risk management; reducing back office costs; and improving their reputations.As a continuation of what was said above, this paper focuses on the reasons why real time data should be used in finance or banking, and in proposing model for banks in Albania. This model is based on the presentation of real time reports to optimize cross selling, customer data quality and credit rating in banks.

\subsection{Concept of Real-time Data Warehouse: Literature review}

Data warehouses (DWs) are the main decision-support assets in many business grounds. Using data mining on DWs enables producing business knowledge. DWs typically store the complete business history, producing decision support information by using On-Line Analytical Processing (OLAP) tools. Extraction, Transformation and Loading (ETL) tools are used for extracting data, transforming and cleansing it into an analytical format, and finally loading it into the DW database. It has been well accepted that DW databases have been updated periodically, typically in a daily, weekly or even monthly basis (Kimball, 2004). Using this update method it is obtained 1) Storage the whole business history, it has huge tables and complex optimization data structures and 2) Business decisions were made on a daily, weekly or monthly basis, dismissing recent real-time business data. This type of update strategy meant that DW data was never up-to-date; transactional data saved between those updates was not included in its databases, excluding the most recent transactional data from OLAP results. A paper by Oracle states that the "Holy Grail" of data warehousing is to support analysis at the speed of the business, affirming the concept of "on-time information" as having information available whenever it is needed (Santos \&Bernardino \&Vieira, 2011). All these requirements are met by real time data warehouse, so decision-making and freshness occurs much more frequently.

In some literatures real time data warehouse is described as new data warehouse architecture which is based on the traditional data warehouse development. Real-time means detect and 


\section{$5^{\text {th }}$ International Conference On Applied Research In SCIENCE, TECHNOLOGY \& KNOWLEDGE}

26-28 February, 2021

Amsterdam, Netherlands

capture the changed data from business systems in time, and load data into the data warehouse. Users can access and query real-time data warehouse to make tactical decision analysis. (fig1)

Fig 1.Real-Time DW architecture

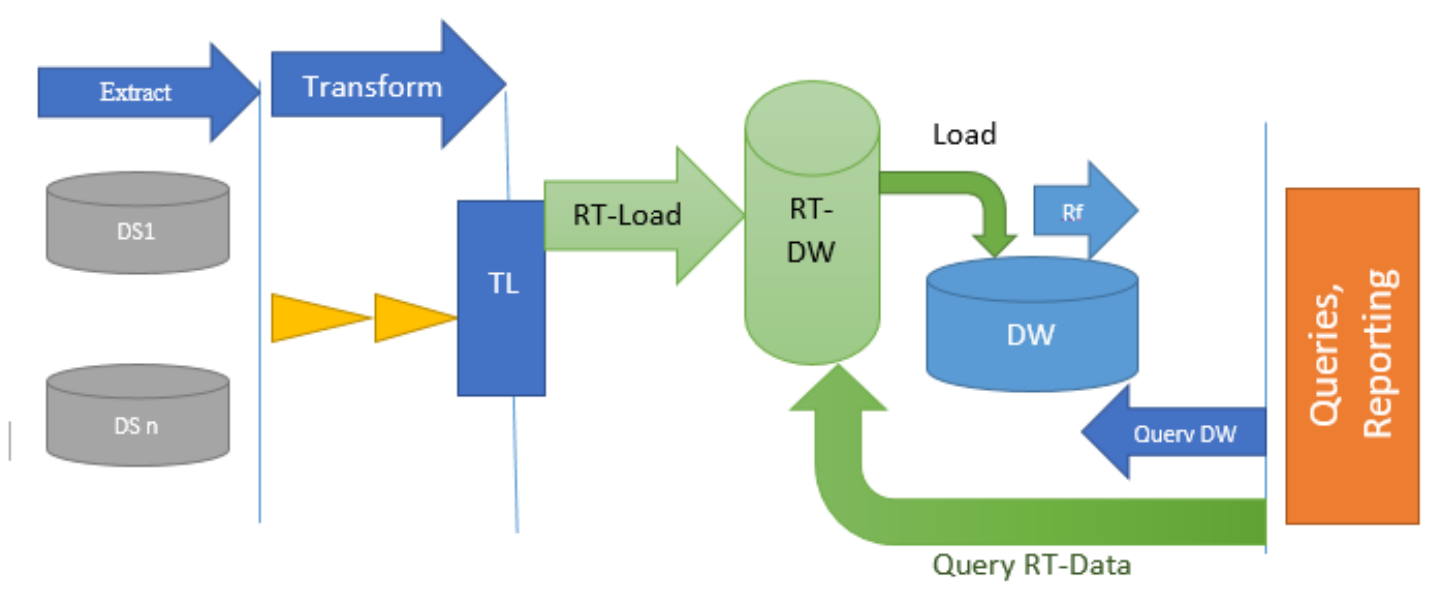

Some research has been done regarding the use of this technology in different fields and according to the requests of users, and among of this on which is presented by T. B. Pedersen. In this report from a knowledge exchange network formed by several major technological partners in Denmark refer that all partners agree real-time enterprise and continuous data availability is considered a short term priority for many business and general data-based enterprises. Nowadays, IT managers are facing crucial challenges deciding whether to build a real-time data warehouse instead of a conventional one and whether their existing data warehouse is going out of style and needs to be converted into a real-time data warehouse to remain competitive (Santos\&Bernardino,2008).

\section{Why should real time data be used in the financial market?}

Large amounts of financial data are generated every second for stock and option trades from several different markets. Apart from being big, this data is also very dynamic and it can be used by companies to detect opportunities and threats and to react towards them quickly. An example of these opportunities consists on predicting upward or downward trends in prices of some securities before a change actually occurs. The most convenient reaction to such opportunity can be buying securities before their prices increase or selling some of them before their prices drop. Afterwards, many of the sold securities can be repurchased at lower prices in order to increase profits. The earlier such decisions are made the higher the chances are of making more profit. In order for these types of opportunities to be successfully seized, good 


\section{$5^{\text {th }}$ International Conference On Applied Research In SCIENCE, TECHNOLOGY \& KNOWLEDGE}

26-28 February, 2021

Amsterdam, Netherlands

forecast models that are based on both current and historical information are needed. When it comes to financial risks, some examples include fraud and criminal activity. The timely identification of the fraudulent and illegal activities of stock markets, such as market manipulation, price fixing, and uninformed inner trading, helps to quickly stop such activities, thereby enhancing market efficiency and securing transactions for investors. Financial opportunities cannot be used and risks cannot be avoided unless the process of detection is very rapid. People may discover these financial opportunities and risks; however, they typically need a long time and the possibilities will no longer be open and the threats will take place before they can avoid them. Automated trading systems have been recently established in order to discover and respond to financial opportunities. Additionally, several real-time fraud detection systems have also been developed to identify and prevent financial threats in a welltimed manner. In order to achieve real time performance, such systems currently rely on smaller and limited data sets. However, for these systems to be more successful they need to deal with all available data. Typically, this financial data is enormous and changes dynamically, which poses many technological challenges for such applications. Traditionally, to uncover any opportunities and risks, Data Mining and Business Intelligence Strategies are primarily used in the financial sector. These types of techniques only operate on historical data and require a high degree of business consumer participation. While these techniques have shown to provide valuable information for the financial sector for long and medium-level business planning, they have very broad detection and response latencies. As a consequence, these strategies cannot be efficiently used for timely detection and prevention processes.

\subsection{An alternative for banking: Toward real-time credit ratings}

Processing data to create credit scores for applicants in real time is an application that is not exclusive to financial markets but has relevance to banking for customers and small and medium enterprises. The timeline of the procedure would be familiar to anyone who has applied for a large amount of credit. Traditionally, banks obtain information from both the application form and other sources regarding the applicant. To develop a credit proposal for the customer, which includes the interest rate and terms of repayment, specialists evaluate this information. There may be some bargaining among the applicant and the bank, including tradeoffs for better overall terms between different loan criteria. The customer will participate in his or her financial operation and further pay off the loan after the credit contract is signed. It not only takes time to collect data and negotiate terms, but these two factors also complicate the picture. First, many of the central data stores used for credit ratings are only published monthly, so the bank might not be aware of the client's recent financial problems from those sources. In addition, non-financial variables may play a role in the risk of default. Several factors related to a large e-commerce platform were examined by the authors such as: frequency of login to the platform, provision of additional contact data (including mobile phone number), amount of transactions in the last month, total number of active transactions, time as a platform user, business sector of the customer, and so on. On historical client results, the authors use linear regression analysis over groups of these parameters to extract a correlation between the 


\section{$5^{\text {th }}$ International Conference On Applied Research In SCIENCE, TECHNOLOGY \& KNOWLEDGE}

26-28 February, 2021

Amsterdam, Netherlands

likelihood of default and the different parameters (Trelewicz, 2017). The effect is a model that could, based on online actions, promote real-time credit ratings for the e-commerce platform. In conventional, central credit-rating databases, such criteria are not even present.

\section{Using real time data process in banks brings these benefits:}

\section{Enhancing the experience of customers}

Much has been written about the millennial generation's rippling influence. Banks are not immune either. In general, this generation, those mostly in their 20s, are tech-savvy, networked, and have high-earning potential. They have been brought up in a real-time environment and they demand better personalized goods and services, greater responsiveness, greater convenience and greater openness. They are likely to be "underbanked," depending on a banking services portfolio that has been stripped down. As a result, the disconnection between their expectations and the banking fact could not have been encountered yet. But they will invariably begin to understand the disconnection, leaving banks to try to keep up with the demands of millennial consumers.

Meanwhile, the banking experience for the wider consumer population could also be enhanced by a real-time model. Banks can process transactions instantly and provide around-the-clock service instead of having clients plan around bank schedules, coping with cut-off times, waiting for transactions to clear, and the like.

\section{Unleashing creativity in goods}

Banks want their customers to have a broad view, including demographics, spending habits, credit card purchases, savings and brokerage accounts, loans, and more. They will methodically pitch goods and services that fit consumer requirements with real-time banking.

Banks can get an up-to-the-minute understanding of consumer needs, build more efficient relationships, and create on-the-spot business opportunities with real-time analytics provided by real-time transactions.

\section{Improving integration across multichannel}

In order to keep up with consumer demands and technological advances, several banks have added new service platforms at a dizzying rate in recent years. Although these platforms are far more advanced than the back-end machinery of a bank, they are basically stand-alone systems, offering inconsistent functionality and details. Regardless of the ability of clients to use these platforms, after-the-fact transactions are always processed, which can create uncertainty.

\section{Strengthening management of fraud}

Real-time banking will help speed up the process of detecting possible fraud and reacting to it. In the current banking environment, on various platforms, online, branch, ATM, point-of-sale, 


\section{$5^{\text {th }}$ International Conference On Applied Research In SCIENCE, TECHNOLOGY \& KNOWLEDGE}

26-28 February, 2021

Amsterdam, Netherlands

transactions can be initiated with each channel interacting with the core network separately. The core network becomes one of the only points of synchronization where banks can detect possible criminal activity, as these networks function independently. Banks can classify fraudulent transactions as they occur by examining aggregate trends using a real-time system, which may also integrate other types of data such as location information.

\section{Informing decisions on risk and compliance}

Because of the complicated patchwork of networks created in an attempt to make a real-time appearance, banks may be challenged to comply with evolving regulations in the industry. Most banks will likely need to develop their risk management and reporting capabilities under regulatory pressure in areas such as fraud detection and customer liquidity. In addition, potential legislation on consumer protection, for example, may require banks to increase transparency and awareness about the real-time financial status of a customer. For instance, if a client is about to commence a transaction that will overdraw an account, the bank can send a text message reminding the client of the possible overdraft (and resulting fees). In this situation, banks not only conform to laws, but also enhance the experience of the consumer. (Johnston, 2012)

\section{A proposal model of using real time for banks in Albania:}

Banks are a complex environment in terms of data management, customer and business requirements. Also increasing their revenue and improving customer retention is a challenge at all the time. Improving the technological infrastructure in banks can be done in several directions, but we have chosen optimizing data warehouse, by using real time reports. (fig 2)

Fig 2.Real Time Reporting System for Banking

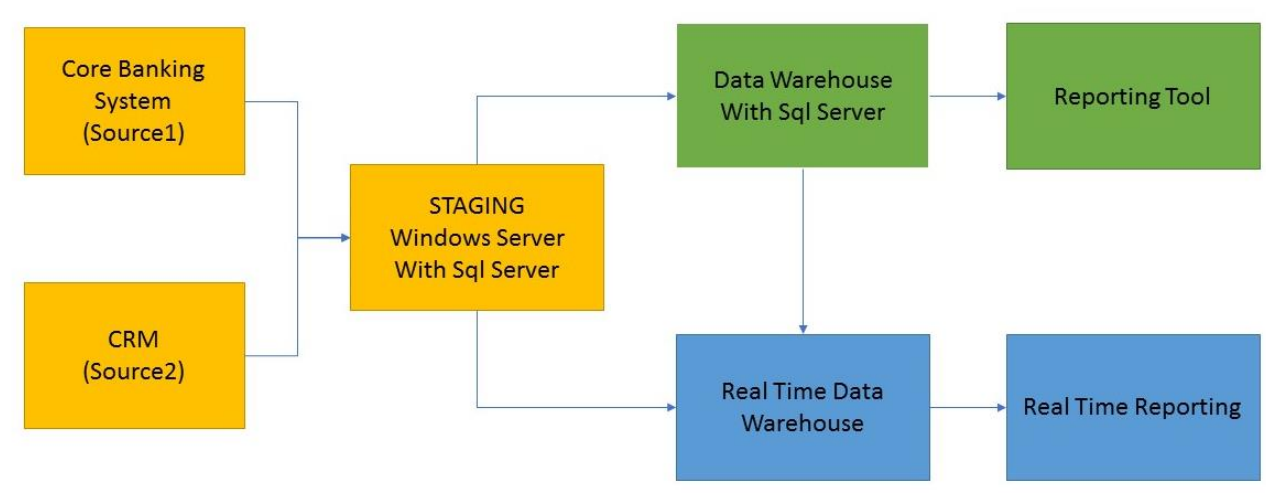




\section{$5^{\text {th }}$ International Conference On Applied Research In SCIENCE, TECHNOLOGY \& KNOWLEDGE}

26-28 February, 2021

Amsterdam, Netherlands

Cross selling Report is a real time report which allows the Cashier or other Bank Employees to sell banking products more easily. By doing an analysis together with the business department in the bank we have identified 6 different types of products that can be sold in this way. Based on the customer data as well as the actions he performs in the bank we manage to analyze and identify these ALERTS FOR SALE and in Real Time there are Alerts for products that can be sold to the requested customer.

For example: Offer e-banking: The offer of this product is based on the number of transactions that the customer makes in the bank, if the number of transactions of the last month is more than 50 transactions then the customer is a potential customer for this product. (Fig1)

Offer Junior Deposit: If the client has a salary account, is married and has 1+ children then he offers to open the Junior Deposit. (Fig 3)

Fig 3.Real time Cross-selling report

\begin{tabular}{|c|c|}
\hline \multicolumn{2}{|c|}{ Cross Selling Tool } \\
\hline Search: Customer Id 1002 & Personal Nr: A61214007B \\
\hline 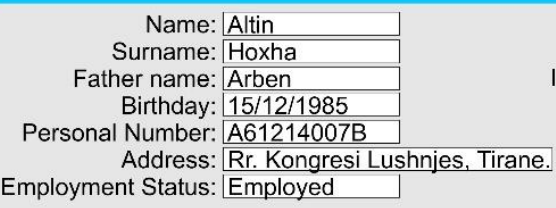 & $\begin{aligned} \text { Mobile: } & +355686868098 \\
\text { Customer Type: } & \text { Individe } \\
\text { Identification Type: } & \text { ID card } \\
\text { Married: } & \text { yes } \\
\text { Sex: } & \text { M } \\
\text { Child Number: } & 2\end{aligned}$ \\
\hline \multicolumn{2}{|c|}{ You should offer to this customer products below: } \\
\hline $\begin{array}{l}\text { Offer Ebanking } \\
\text { Offer Payroll account } \\
\text { Offer Fixed Time Deposit }\end{array}$ & $\begin{array}{l}\text { Offer Debit card } \\
\text { Offer Junior Deposit } \\
\text { Offer Cosumer Loan }\end{array}$ \\
\hline
\end{tabular}

Below is the query that enables the execution of the cross-seller report in the Reporting server, which receives the data from the DW of the bank: 


\section{$5^{\text {th }}$ International Conference On Applied Research In SCIENCE, TECHNOLOGY \& KNOWLEDGE}

26-28 February, 2021 Amsterdam, Netherlands

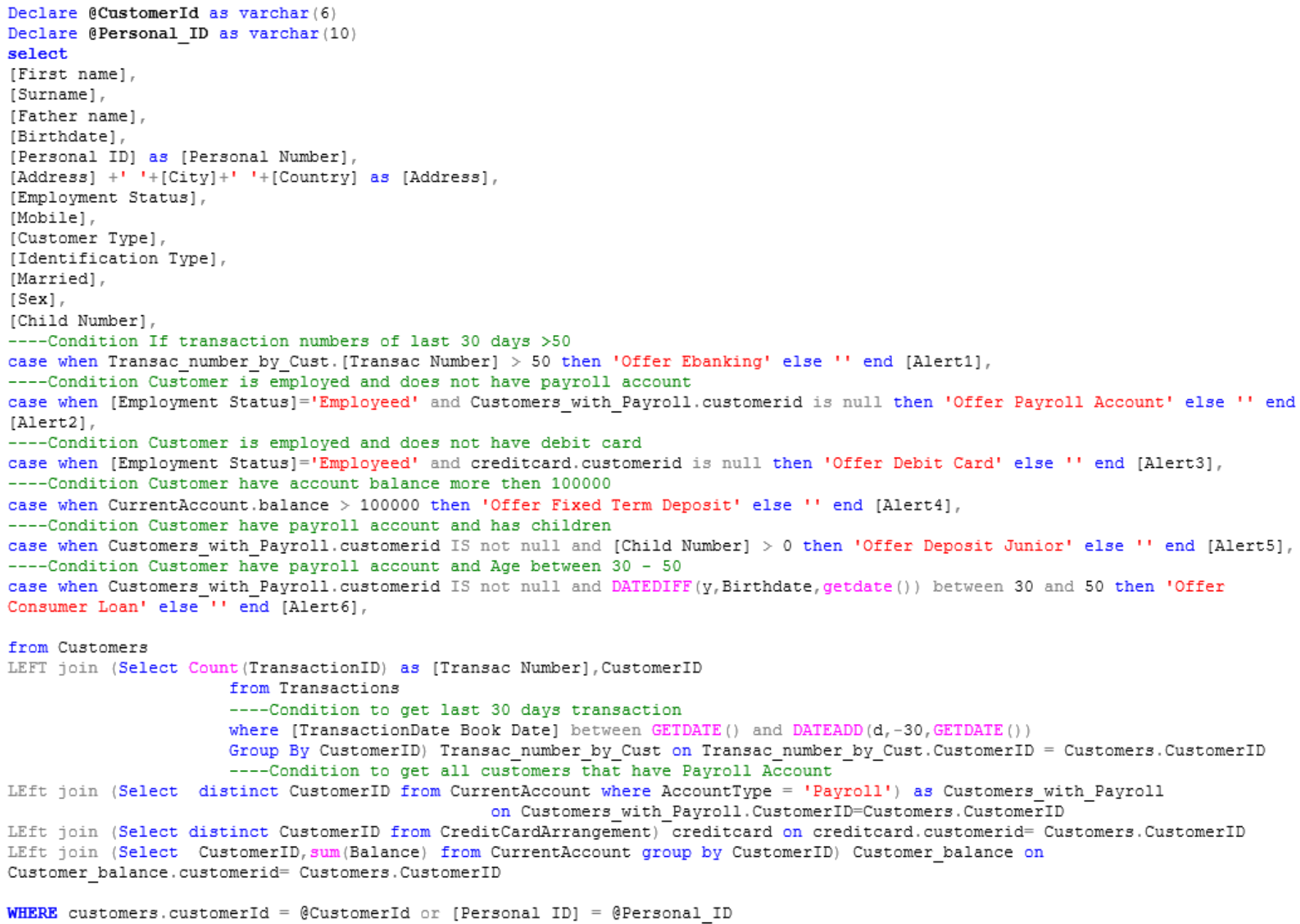

\section{Conclusion}

In a seemingly short time, the use of real-time data to make operational decisions and deliver enhanced customer services has moved from an irregularly used, value-added option to a competitive necessity. Our vision of real-time reporting for banking industry proposes the use of real time querying, in order to eliminate performance problems and to solve problems like internal inconsistency, scalability and data latency.

Regardless of the benefits of using the real time process, has no widespread use in banks, probably because it requires additional infrastructure, additional charge. Cross selling and credit ratings are among the main processes of the bank, and optimizing them using real time data makes a great business sense for banks. Using the model proposed in this paper, banks and other financial enterprises can make better offers to the customer in a timely manner and can certainly sell more. Financial services institutions now have the opportunity to combine their institutional heft with the latest technologies, delivering the latest and greatest services cost-effectively. 


\section{$5^{\text {th }}$ International Conference On Applied Research In SCIENCE, TECHNOLOGY \& KNOWLEDGE}

26-28 February, 2021

Amsterdam, Netherlands

In future works we will implement a model for credit rating improvement through real time data reporting.

\section{References}

R. J. Santos, J. Bernardino and M. Vieira., (2011)."24/7 Real-Time Data Warehousing: A Tool for Continuous Actionable Knowledge," 2011 IEEE 35th Annual Computer Software and Applications Conference, Munich, pp. 279-288, doi:10.1109/COMPSAC.2011.44.

Kimball, R., Caserta, J. (2004): The Data Warehouse ETL Toolkit, Wiley Computer Pub.

Santos, R. J. and J. Bernardino. (September 2008) "Real-time data warehouse loading methodology." IDEAS '08 (2008).Proceedings of the 2008 international symposium on Database engineering \& applications Pages 49-58 https://doi.org/10.1145/1451940.1451949

J. Q. Trelewicz. (2017) "Big Data and Big Money: The Role of Data in the Financial Sector," in IT Professional, vol. 19, no. 3, pp. 8-10, 2017, doi: 10.1109/MITP.2017.45.

Brian Johnston, (March 2012) .6 Business Benefits of Real-Time Banking. The Wall Street Journal. Available: https://deloitte.wsj.com/cio/2012/03/29/six-business-benefits-of-realtime-banking/ 